\title{
Complexity of Consistent Query Answering in Databases under Cardinality-Based and Incremental Repair Semantics
}

\author{
Andrei Lopatenko* \\ Free University of Bozen-Bolzano \\ Faculty of Computer Science \\ Bozen-Bolzano, Italy. \\ lopatenko@inf.unibz.it
}

\author{
Leopoldo Bertossi \\ Carleton University \\ School of Computer Science \\ Ottawa, Canada. \\ bertossi@scs.carleton.ca
}

\begin{abstract}
Consistent Query Answering (CQA) is the problem of computing from a database the answers to a query that are consistent with respect to certain integrity constraints that the database, as a whole, may fail to satisfy. Consistent answers have been characterized as those that are invariant under certain minimal forms of restoration of the database consistency. In this paper we investigate algorithmic and complexity theoretic issues of CQA under database repairs that minimally depart -wrt the cardinality of the symmetric difference- from the original database. Research on this kind of repairs has been suggested in the literature, but no systematic study had been done. Here we obtain first tight complexity bounds. We also address, considering for the first time a dynamic scenario for CQA, the problem of incremental complexity of CQA, that naturally occurs when an originally consistent database becomes inconsistent after the execution of a sequence of update operations. Tight bounds on incremental complexity are provided for various semantics under denial constraints, e.g. (a) minimum tuple-based repairs wrt cardinality, (b) minimal tuple-based repairs wrt set inclusion, and (c) minimum numerical aggregation of attribute-based repairs. Fixed parameter tractability is also investigated in this dynamic context, where the size of the update sequence becomes the relevant parameter.
\end{abstract}

\section{Introduction}

Integrity constraints (ICs) capture the semantics of data and are expected to be satisfied by a database in order to keep its correspondence with the outside reality it is modelling. However, it is often the case that IC satisfaction cannot be guaranteed, and inconsistent database states are common, e.g. in integrated databases, census databases, legacy data, etc. [7].

Consistent Query Answering (CQA) is the problem of computing from a database those answers to a query that are consistent with respect to certain ICs, that the database as a whole may fail to satisfy. Consistent answers have been characterized as those that are invariant under minimal forms of restoration of the database consistency [2]. From this perspective, CQA is a form of cautious reasoning from a database under integrity constraints.

The notion of minimal restoration of consistency was captured in [2] in terms of database repairs, i.e. new, consistent database instances that share the schema

\footnotetext{
* Also: University of Manchester, Department of Computer Science, UK.
} 
with the original database, but differ from the latter by a minimal set of whole tuples under set inclusion. In $[7,19,2,9,4,11]$ complexity bounds for CQA under this repair semantics have been reported. However, less attention has received the semantics of CQA based on "cardinality-based repairs" of the original database that minimize the number of whole database tuples by which the instances differ.

Example 1. Consider a database schema $P(X, Y, Z)$ with the functional dependency $X \rightarrow Y$. The inconsistent instance $D=\{P(a, b, c), P(a, c, d), P(a, c, e)\}$, seen as a set of ground atoms, has two repairs wrt set inclusion, namely $D_{1}=$ $\{P(a, b, c)\}$ and $D_{2}=\{P(a, c, d), P(a, c, e)\}$, because the symmetric set differences with the original instance, i.e. $\Delta\left(D, D_{1}\right), \Delta\left(D, D_{2}\right)$, are minimal under set inclusion. However only $D_{2}$ is a cardinality-based repair, because the cardinality $\left|\Delta\left(D, D_{2}\right)\right|$ of the symmetric set difference becomes a minimum.

In this paper we address the problem of obtaining complexity bounds for CQA under the semantics given by cardinality-based repairs, and we do this by introducing some graph theoretic techniques and results that, apart from being interesting by themselves, have a wider applicability in the context of CQA. Although research on cardinality- and tuple-based repairs has been proposed and started before in the context of CQA [7], no detailed analysis of their complexity theoretic properties has been provided. In [3] a brief illustration was given of how to specify cardinality based repairs using logic programs with weak cardinality constraints [8] and stable model semantics.

Our emphasis is on CQA, as opposed to computing or checking repairs. This is because we are usually not interested in computing specific repairs (there are exceptions though, e.g. in census-like data [6]), but in characterizing and computing consistent answers to queries. However, the repair semantics we choose will have an impact on CQA.

Example 2. (example 1 continued) The query $P(x, y, x)$ ? has $(a, c, d)$ and $(a, c, e)$ as consistent answers under the cardinality semantics (the classic answers in the only repair), but none under the set inclusion semantics (there is no classic answer shared by the two repairs).

All the complexity bounds on CQA given so far in the literature, no matter what repair semantics is chosen, consider the static case: Given a snapshot of a database, a set of integrity constraints, and a query, the problem is to find consistent query answers. However, databases are essentially dynamic structures, subject to update operations. In this paper we also take into account dynamic aspects of data, studying the complexity of CQA when the consistency of a consistent database may be affected by update actions.

Example 3. (examples 1 and 2 continued) The cardinality-based repair $D_{2}=$ $\{P(a, c, d), P(a, c, e)\}$ is obviously consistent, however after the execution of the update operation insert $(P(a, f, d))$ it becomes inconsistent. In this case, the only cardinality repair of $D_{2} \cup\{P(a, f, d)\}$ is $D_{2}$ itself. So, CQA from $D_{2} \cup\{P(a, f, d)\}$ amounts to classic query answering from $D_{2}$. However, if we start from the consistent instance $D^{\prime}=\{P(a, c, d)\}$, executing the same update operation leads to two cardinality repairs, namely $D^{\prime}$, but also $\{P(a, f, d)\}$, and now CQA from $D^{\prime} \cup\{P(a, f, d)\}$ is different from classic query answering from $D^{\prime}$, because two repairs have to be taken into account. 
In this case, it would be inefficient to compute a materialized repair of the database or a consistent answer to the query from scratch after every update. In this paper we investigate how a pre-computed repair of the database at a previous step or the original instance itself if it was already consistent can be used to consistently answer queries after update operations. We provide a unified approach to the study of the computational complexity of incremental consistent query answering; and not only under cardinality-based repairs, but also under other repair semantics, like the classic minimal set inclusion semantics and the one based on minimization of changes of attribute values (attribute-based repairs) that have been already used in the literature [35, 18, 6, 17].

Incremental algorithms have been developed to check integrity constraint satisfaction [28]. In a similar spirit we find work on incremental database maintenance, i.e. integrity constraint satisfaction, by means of compensating active rules [34]. However, to the best of our knowledge, incremental CQA and incremental repair computation (under updates) have not been treated before. We know, by data complexity theoretic reasons, that some first-order queries asking for consistent answers cannot be expressed as first-order queries asking for classic answers $[7,9,11,19]$. However, in the incremental, dynamic context consistent answers could be expressed as classic answers to first-order queries. A similar situation can be found in incremental evaluation of queries that, statically, are not expressible in the query language at hand, but incremental computation can be performed and expressed [26].

Cardinality-based CQA as studied in this paper has interesting properties that make it useful as a semantics for CQA. First of all, as illustrated in Example 1 , it is clearly the case that every cardinality-based repair is also a set inclusion-based repair, but not necessarily the other way around. In consequence, the consistent query answers under cardinality repairs form a superset of the consistent answers under the set inclusion-based semantics. Actually, in situations where the latter does not give any answers (c.f. Example 2), the former does return answers, which is good. They could be further filtered out according to other criteria at a post-processing phase. In extreme cases, when there is only one database tuple in semantic conflict with the rest of a possible large set of other tuples, the existence of a set inclusion-based repair containing the only conflicting tuple would easily lead to an empty set of consistent answers. The cardinality-based semantics would not allow such a repair. (Example 4 below illustrates this situation.)

This feature of the cardinality-based repair semantics comes at a price. In Section 3 we prove that CQA has a higher data complexity than the classic, set inclusion-based semantics, actually $P^{N P(\log (n))}$-hard vs. PTIME for denial constraints [11]. On the other side, the cardinality-based semantics has the interesting property that CQA, a form of cautious (or certain) reasoning (true in all repairs) and its brave (or possible) version, i.e. true in some repair, are mutually poly-time reducible and share the same complexity. This is established in Section 3 by proving first some useful graph theoretic lemmas about maximum independent sets. This result may not hold for classic CQA.

Furthermore, we prove in Sections 4.1 and 5 that incremental CQA for conjunctive queries under the cardinality-based semantics has a lower complexity than incremental CQA for the classic semantics, actually PTIME vs. coNP-hard 
(in data complexity), which makes the cardinality semantics more appealing in a dynamic setting.

As we just mentioned, incremental CQA under the cardinality semantics is polynomial in data complexity, but naive algorithms are exponential if the size of the update sequence is a part of the input and the combined complexity is considered. In Section 4.1 we study the parameterized complexity [15, 22] of CQA, actually for the incremental case and under cardinality-based semantics, where the parameter is the size of the update sequence. We establish that the problem is fixed parameter tractable by providing a concrete parameterized algorithm.

For comparison with the cardinality-based semantics, we obtain in Section 5 new results on the static and incremental complexity under the classic semantics (i.e. tuple- and set inclusion-based distance) and the attribute-based semantics (i.e. attribute value-based and minimization of attribute changes). We prove that static CQA for the weighted version of the attribute-based semantics and incremental CQA under the attribute-based semantics become both $P^{N P}$-hard in data.

We concentrate on relational databases and basically on the class of denial integrity constraints, which includes most of the constraints found in applications where inconsistencies naturally arise, e.g. census-like databases [6], experimental samples databases, biological databases, etc. Complexity results refer to data complexity [1]. For complexity theoretic definitions and classic results we refer to [31], to [1] for foundations of databases, and to [15] for parameterized complexity.

\section{Preliminaries}

A relational database $D$ can be identified with a finite set of ground atoms of the form $R(\bar{t})$, where $R$ is a relation in the database schema $\mathcal{D}$, and $\bar{t}$ is a finite sequence of constants taken from the underlying database domain $\mathcal{U}$. The ground atom $R(\bar{t})$ is also called a database tuple. ${ }^{1}$ The relational schema $\mathcal{D}$ determines a first-order language $L(\mathcal{D})$ based on the relation names, the elements of $\mathcal{U}$, and extra built-in predicates. In the language $L(\mathcal{D})$, integrity constraints are sentences, and queries are formulas, usually with free variables. We assume in this paper that sets $I C$ of ICs are always consistent in the sense that they are simultaneously satisfiable as first-order sentences. A database is consistent wrt to a given set of integrity constraints $I C$ if the sentences in $I C$ are all true in $D$, denoted $D \models I C$. An answer to a query $Q(\bar{x})$, with free variables $\bar{x}$, is a tuple $\bar{t}$ that makes $Q$ true in $D$ when the variables in $\bar{x}$ are interpreted as the corresponding values in $\bar{t}$, denoted $D \models Q[\bar{t}]$.

For a database $D$, possibly inconsistent with respect to $I C$, the consistent answers to a query $Q$ from $D$ wrt $I C$ are characterized as those answers that are invariant under all minimal forms of restoration of consistency for $D$, where minimality refers to some sort of distance between the original instance $D$ and alternative consistent instances.

\footnotetext{
${ }^{1}$ We also use the term tuple to refer to a finite sequence $\bar{t}=\left(c_{1}, \ldots, c_{n}\right)$ of constants of the database domain $\mathcal{U}$, but a database tuple is a ground atomic sentence with predicate in $\mathcal{D}$ (excluding built-ins predicates, like comparisons).
} 
Definition 1. For a database $D$, integrity constraints $I C$ and a partial order $\preceq_{D, \mathcal{S}}$ over databases depending on the original database $D$ and a repair semantics $\mathcal{S}$, a repair of $D$ wrt $I C$ under $\mathcal{S}$ is an instance $D^{\prime}$ such that: (a) $D^{\prime}$ has the same schema and domain as $D$; (b) $D^{\prime} \models I C$; and (c) there is no $D^{\prime \prime}$ satisfying (a) and (b), such that $D^{\prime \prime} \prec_{D, \mathcal{S}} D^{\prime}$, i.e. $D^{\prime \prime} \preceq_{D, \mathcal{S}} D^{\prime}$ and not $D^{\prime} \preceq_{D, \mathcal{S}} D^{\prime \prime}$. The set of all repairs is denoted with $\operatorname{Rep}(D, I C, \mathcal{S})$.

The class $\operatorname{Rep}(D, I C, \mathcal{S})$ depends upon the semantics $\mathcal{S}$, which determines the partial order $\preceq$ and the way repairs can be obtained, e.g. by allowing both insertions and deletions of whole database tuples [2], or deletions of them only [11], or only changes of attribute values [35, 6, 17], etc. (c.f. Definition 3.)

Definition 2. Let $D$ be a database, $I C$ a set of ICs, and $Q(\bar{x})$ a query. (a) A ground tuple $\bar{t}$ is a consistent answer to $Q$ wrt $I C$ under semantics $\mathcal{S}$ if for every $D^{\prime} \in \operatorname{Rep}(D, I C, \mathcal{S}), D^{\prime} \models Q[\bar{t}] . \quad($ b) $C q a(Q, D, I C, \mathcal{S})$ is the set of consistent answers to $Q$ in $D$ wrt $I C$ under semantics $\mathcal{S}$. If $Q$ is a sentence (a boolean query), $C q a(Q, D, I C, \mathcal{S}):=\{$ yes $\}$ when $D^{\prime} \models Q$ for every $D^{\prime} \in \operatorname{Rep}(D, I C, \mathcal{S})$, and $C q a(Q, D, I C, \mathcal{S}):=\{n o\}$, otherwise. (c) $C Q A(Q, I C, \mathcal{S}):=\{(D, \bar{t}) \mid \bar{t} \in$ $C q a(Q, D, I C, \mathcal{S})\}$, the decision problem of consistent query answering.

The decision problem of CQA just defined if for the static case, in the sense that it only considers a snapshot of the database. In the literature different notions of distance have been considered, they give rise to different repair semantics. We summarize here the most common ones, those that will be investigated in this work. In the following, $\Delta\left(D^{\prime}, D\right)$ denotes the symmetric difference $\left(D^{\prime} \backslash D\right) \cup$ $\left(D \backslash D^{\prime}\right)$ of two database instances conceived both as set of ground atoms.

Definition 3. (minimality semantics) (a) Minimal set inclusion semantics (simply, S-repair semantics) [2]: $D^{\prime} \preceq D^{\prime \prime}: \Longleftrightarrow \Delta\left(D^{\prime}, D\right) \subseteq \Delta\left(D^{\prime \prime}, D\right)$. (b) Minimum cardinality set semantics (C-repair semantics): $D^{\prime} \preceq D^{\prime \prime}: \Longleftrightarrow\left|\Delta\left(D^{\prime}, D\right)\right| \leq$ $\left|\Delta\left(D^{\prime \prime}, D\right)\right|$. (c) Aggregate attribute difference semantics (A-repair semantics) minimizes a numerical aggregation function over attribute changes throughout the database.

Particular classes of A-repairs can be found in $[18,17]$, where the aggregation function to be minimized is the number of all attribute changes; and in [6], where the function is the overall quadratic difference obtained from the changes in numerical attributes between the original database and the repair.

S-repairs and C-repairs are examples of tuple-based repairs, in the sense that consistency is restored by inserting and/or deleting database tuples. A-repairs are attribute-based repairs, under which database instances can be repaired by changing attributes values in existing tuples only. Classes of attribute-based repairs have been studied in $[35,18,6,17]$. Another notion of attribute-based repair, not explored so far, and not included in Definition 3, could minimize, settheoretically, the set of attribute changes, with priorities imposed on attributes. We will consider other repair semantics later on (e.g. Definition 5) and particular cases of attribute-based repairs (c.f. Section 5.2).

It is easy to prove that every C-repair is an S-repair; and consequently every consistent query answer under the S-semantics is a consistent query answer under 
the C-semantics. However, as Example 1 shows, not every S-repair is an Crepair. In that example, attribute-based repairs could be $\{P(a, c, c), P(a, c, d)$, $P(a, c, e)\}$, suggesting that we we made a mistake in the second argument of the first tuple, but also $\{P(a, b, c), P(a, b, d), P(a, b, e)\}$. If the aggregate function in Definition $3(\mathrm{c})$ is the number of changes in attribute values, the former would be a repair, but not the latter. These instances are neither S- nor C-repairs if the changes of attribute values have to be simulated via deletions followed by insertions.

Integrity constraints may be any first-order sentences written in language $L(\mathcal{D})$, but most of our results refer to denial constraints only.

Definition 4. Denial constraints are integrity constraints of the form $\forall \bar{x} \neg\left(A_{1} \wedge\right.$ $\ldots \wedge A_{m} \wedge \gamma$ ), where each $A_{i}$ is a database atom and $\gamma$ is a conjunction of comparison atoms.

Notice that functional dependencies (FDs), e.g. $\forall x \forall y \forall z \neg(R(x, y) \wedge R(x, z) \wedge y \neq$ $z)$, are binary denial constraints; and range constraints are one-database atom denials. For denial ICs, tuple-based repairs are obtained by tuple deletions only [11].

In this paper we concentrate on data complexity. We briefly recall some of the complexity classes used in this paper. $F P$ is a class of functional problems associated with languages in the class $P$ of decision problems that are solvable in polynomial time. $P^{N P}$ (or $\Delta_{2}^{P}$ ) is the class of decision problems solvable in polynomial time by a machine that makes calls to an $N P$ oracle. $P^{N P(\log (n))}$ is similarly defined, but the number of calls is logarithmic. It is not known if $P^{N P(\log (n))}$ is strictly contained in $P^{N P}$. The functional class $F P^{N P(\log (n))}$ is similarly defined. The class $\Delta_{3}^{P}(\log (n))$ contains decision problems that can be solved by a polynomial time machine that makes a logarithmic number of calls to an oracle in $\Sigma_{2}^{P}$.

\section{Complexity of CQA under the C-Repair Semantics}

CQA under the minimal cardinality repair semantics (C-repair semantics in Definition 3(b)) has received less attention in the literature than the same problem under the S-repair semantics. An exception is [3], where C-repairs were specified using logic programs with non-prioritized weak constraints under the skeptical stable model semantics [8]. As a consequence, from results in [8] (c.f. also [25]), we obtain that an upper bound on the data complexity of CQA under the Crepair semantics is the class $\Delta_{3}^{P}(\log (n))$. In this section we investigate the static complexity of tuple-based CQA under the C-repair semantics.

In [4], conflict graphs were first introduced to study the complexity of CQA for aggregate queries wrt FDs under the S-repair semantics. They have as vertices the database tuples and edges connect two tuples that simultaneously violate a FD. There is a one-to-one correspondence between S-repairs of the database and the set-theoretically maximal independent sets (simply called maximal independent sets) in the conflict graph. Similarly, there is a one-to-one correspondence between C-repairs and maximum independent sets in the same graph (but now they are maximum in cardinality). 
Notice that, unless an IC forces a particular tuple not to belong to the database $^{2}$, every tuple in the original database belongs to some S-repair, but not necessarily to a C-repair (c.f. Example 1, where the tuple $P(a, b, c)$ does not belong to the only $\mathrm{C}$-repair). In consequence, testing membership of vertices to some maximum independent set becomes a problem that is relevant to address. For this purpose we will make good use of some graph theoretic constructions and results about maximum independent sets obtained from them, whose proofs use a self-reducibility property of independent sets that can be expressed as follows: For any graph $G$ and vertex $v$, every maximum independent set that contains $v$ (meaning maximum among the independent sets that contain $v$ ) consists of vertex $v$ together with a maximum independent set of the graph $G^{\prime}$ that is obtained from $G$ by deleting all vertices adjacent to $v$.

Lemma 1. Given a graph $G$ and a vertex $v$ in it, a graph $G^{\prime}$ that extends $G$ can be constructed in polynomial time in the size of $G$, such that there is a maximum independent set $I$ of $G$ containing $v$ iff $v$ belongs to every maximum independent set of $G^{\prime}$ iff the sizes of maximum independent sets in $G$ and $G^{\prime}$ differ by one.

Actually the graph $G^{\prime}$ in this lemma can be obtained by adding a new vertex $v^{\prime}$ that is connected only to the neighbors of $v$. Conversely, the following holds

Lemma 2. For every graph $G$ and vertex $v$ there is a graph $G^{\prime}$ that can be constructed in polynomial time in the size of $G$, such that $v$ belongs to all maximum independent sets of $G$ iff $v$ belongs to some maximum independent set of $G^{\prime}$.

From the lemmas and the membership to $F P^{N P(\log (n))}$ of computing the size of a maximum clique in a graph [24], we obtain

Proposition 1. The problems of deciding for a vertex in a graph if it belongs to some maximum independent set and if it belongs to all maximum independent set are both in $P^{N P(\log (n))}$.

Since a ground atomic query is consistently true when it belongs, as a database tuple, i.e. as a vertex in the conflict graph, to all the maximum independent sets of the conflict graph, we obtain

Corollary 1. For functional dependencies and ground atomic queries, CQA under the C-repair semantics belongs to $P^{N P(\log (n))}$.

Considering the maximum independent sets (or the C-repairs) as a collection of possible worlds, the previous lemmas show a close connection between the certain and possible C-semantics, that sanctions something as true if it is true in every (the default for CQA), resp. some possible world. CQA under these semantics and functional dependencies are polynomially reducible to each other, and also share the same complexity.

Using this result, it is possible to extend Corollary 1 to negative atomic queries because $P^{N P(\log (n))}$ is closed under complement: Notice that a vertex

\footnotetext{
${ }^{2}$ We do not consider in this work such non generic ICs [7].
} 
does not belongs to any maximum independent sets means that the certain answer to the corresponding negated query is yes and the possible answer to the corresponding atomic query is no (or better, false in the sense that it is false in every C-repair). On the other side, that there is a maximum independent set to which a vertex does not belong means that the possible answer to the corresponding negated query is yes and the certain answer to the positive query is no. Corollary 1 also holds for queries that are conjunctions of atoms.

The next result shows that graphs with their maximum independent sets can be uniformly encoded as database repair problems under the C-semantics.

Proposition 2. There is a fixed database schema $\mathcal{D}$ and a denial constraint $\varphi$ in $L(\mathcal{D})$, such that for every graph $G$, there is an instance $D$ over $\mathcal{D}$, whose Crepairs wrt $\varphi$ are in one-to-one correspondence with the maximum independent sets of $G$. Furthermore, $D$ can be built in polynomial time in the size of $G$.

This proposition is a representation result, of the maximum independent sets of a graph as the C-repairs of an inconsistent database wrt a denial constraint. This is interesting, because conflict graphs for databases wrt denial constraints are actually conflict hypergraphs [11] that have as vertices the database tuples, and as hyperedges the (set theoretically minimal) collections of tuples that simultaneously violate one of the denial constraints.

The correspondence for conflict graphs between repairs and independent sets -maximum or maximal depending on the semantics- still holds for hypergraphs, where an independent set in an hypergraph is a set of vertices that does not contain any hyperedges [11]. Lemmas 1 and 2 and Proposition 1 still hold for hypergraphs, and in consequence the polynomial time mutual reducibility between the certain and possible semantics for CQA still holds for denial constraints and ground atomic queries.

From Proposition 2 and the $P^{N P(\log (n))}$-completeness of determining the size of a maximum clique [24], we obtain

Corollary 2. Determining the size of a C-repair for denial constraints is complete for $P^{N P(\log (n))}$.

Using the hypergraph representation of C-repairs, it is possible to generalize Corollary 1 to the case of denial constraints and queries that are conjunctions of atoms.

Proposition 3. For denial constraints and non-existentially quantified conjunctive queries, CQA under the C-repair semantics belongs to $P^{N P(\log (n))}$.

This result can be generalized to conjunctive queries containing negation, but no quantifiers. For example, the query $Q=A_{1} \wedge \cdots \wedge A_{i} \wedge \neg A_{i+1} \cdots \wedge \neg A_{k}$, is true iff each positive conjunct is contained in every maximum independent set, and each atom preceded by a negation is not contained in any maximum independent sets. Deciding if a database tuple $A$ is not contained in any maximum independent sets of a graph $G$ is in $F P^{N P(\log (n))}$, because it is the complement to the problem of deciding if a database tuple $A$ is contained in some maximum independent sets of a graph, and $F P^{N P(\log (n))}$ is closed under complement. 
In order to obtain hardness for CQA under the C-repair semantics, we need a useful graph theoretic construction, the block $B_{k}(G, \mathbf{t})$ (c.f. Figure 1), consisting of two copies $G_{1}, G_{2}$ of $G$, and two internally disconnected subgraphs $I_{k}, I_{k+1}$, with $k$ and $k+1$ vertices, resp. Every vertex in $G\left(G^{\prime}\right)$ is connected to every vertex in $I_{k}$ (resp. $\left.I_{k+1}\right)$.

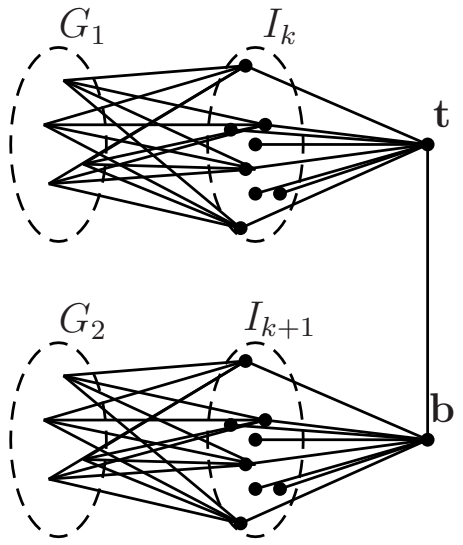

Figure 1. The block $B_{k}(G, t)$

Lemma 3. (the block construction) Given a graph $G$ and a number $k$, there exists a graph $B_{k}(G, \mathbf{t})$, where $\mathbf{t}$ is a distinguished vertex in it, such that $\mathbf{t}$ belongs to all maximum independent sets of $B_{k}(G, \mathbf{t})$ iff the cardinality of a maximum independent set of $G$ is equal to $k$. $B_{k}(G, \mathbf{t})$ can be computed in polynomial time in the size of $G$.

Proposition 4. Deciding if a vertex belongs to all maximum independent sets of a graph is $P^{N P(\log (n))}$-hard.

This result can be proved by reduction from the following $P^{N P(\log (n))}$-complete decision problem [24]: Given a graph $G$ and an integer $k$, is the size of a maximum clique in $G$ equivalent to $0 \bmod k$ ? $G$ is reduced to a graph that is built by combining a number of versions of the block construction in Figure 1. Now, using Proposition 2, the graph constructed for the reduction in Proposition 4 can be represented as a database consistency problem, and in this way we obtain

Theorem 1. For denial constraints, CQA for ground atomic queries under the C-repair semantics is $P^{N P(\log (n))}$-complete.

This theorem is interesting, because CQA for denial constraints, but S-semantics is in PTIME for arbitrary ground atomic queries [11]; and also because query answering under the S-semantics in the context of belief revision/update is more complex than the same problem for C-semantics (assuming the polynomial hierarchy does not collapse); more precisely Winslett's framework [12] (based on set inclusion) is $\Pi_{2}^{P}$-complete, while Dalal's [13] (based on set cardinality) is $P^{N P(\log (n))}$-complete [16]. Connections between CQA and belief revision were already established in [2]. Notice that our complexity results do not follow, at least not straightforwardly from the results for belief revision presented in [16]. They apply in the propositional setting, in combined complexity (as opposed to data complexity), and the revision formulas (in our case the constraints) and the query do not necessarily satisfy our conditions.

Now we consider a weighted version of the C-semantics. Under denial constraints, this means that it may be more costly to remove certain tuples than others to restore consistency. 
Definition 5. (weC-semantics) Assume that every database tuple $R(\bar{t})$ in $D$ has an associated numerical cost $w(R(\bar{t})) . D^{\prime}$ is a repair of $D$ under the weighted minimum cardinality set semantics if the order relation used in Definition 1 is given by $D_{1} \preceq_{D, w} D_{2}: \Longleftrightarrow\left|D \triangle D_{1}\right|_{w} \leq\left|D \triangle D_{2}\right|_{w}$, where $|S|_{w}$ for a set of database tuples $S$ is the sum of the weights of the elements of $S$.

This semantics is a generalization of the C-semantics, that can be obtained by defining all the weights as 1 ; and as such, it is still tuple-based, actually tuple deletion-based for denial constraints.

Proposition 5. CQA for ground atomic queries wrt denial constraints under the weC-repair semantics belongs to $P^{N P}$.

\section{Incremental Complexity of CQA}

We will consider the following problem of incremental CQA: Assume that we have a consistent database $D$ wrt to certain integrity constraints. After an update sequence $U$ on $D$ composed of update operations of any of the forms insert $(R(\bar{t}))$, delete $(R(\bar{t}))$, meaning insert/delete tuple $R(\bar{t})$ into/from $D$, or change $(R(\bar{t}), A, a)$, for changing value of attribute $A$ in $R(\bar{t})$ to $a$, with $a \in \mathcal{U}$, we may obtain an inconsistent database. We are interested in whether we can find consistent query answers from the updated inconsistent database more efficiently by taking into account the previous consistent database state. We will see that for a few particular cases of the consistent query answering problem, the knowledge of the previous state may significantly simplify consistent query answering, while in other cases the worst-case computational complexity of query answering is the same as in the classic, static scenario, where no updates are considered.

Definition 6. For a set of integrity constraints $I C$, a database $D$ that is consistent wrt $I C$, and a sequence $U$ of one-tuple database update operations $U_{1}, \ldots, U_{m}$, incremental consistent query answering for query $Q$ is CQA for $Q$ wrt $I C$ from the instance $U(D)$ that results from applying $U$ to $D$. The complexity of incremental CQA is measured wrt the size of $D$.

In order not to add complexity on the sole basis of the length of the update sequence, we will usually assume that $m$ is small in comparison to the size of the underlying database $D$, say $m<c \cdot|D|$. We are in general interested in data complexity, i.e. wrt $|D|$. However, in Section 4.1 we consider parameterized complexity, where the role of parameter $m$ is considered. Furthermore, we consider an update sequence $U$ as atomic in the sense that it is completely executed or not. In particular, this allows us to concentrate on "minimized" versions of update sequences, e.g. containing only insertions and/or attribute changes when dealing with denial constraints, because deletions do not have any effects on them.

A notion of incremental complexity has been introduced in [27], and also in [23] under the name of dynamic complexity. However, our notion is different. In those papers, the instance that is updated can be arbitrary, and the question is about the complexity for the updated version when information about the 
previous instance can be used. In our case, we are assuming that the initial database is consistent, and then the problem of finding its repairs is trivial (the only repair is the database itself) and CQA is easy (just query the database as usual). However, the same problems for the updated database are not necessarily trivial or easy. Furthermore, as opposed to [27, 23], where new incremental or dynamic complexity classes are introduced, we appeal to those classic complexity classes found at a low level in the polynomial hierarchy, which are applied to data complexity, relative to the size of the initial database.

\subsection{Incremental complexity: C-repair semantics}

In this section we study the computational complexity of CQA under the Csemantics over an inconsistent database that is obtained trough a short sequence of update operations on a consistent database.

Proposition 6. Under the C-semantics, incremental CQA for first-order boolean queries, denial constraints, and a sequence of atomic updates $U: U_{1}, \ldots, U_{m}$ applied to a database $D$ is in PTIME in the size of $D$.

As we saw in Theorem 1, static CQA for denial constraints under the C-semantics is a hard problem, in contrast to incremental CQA for the same class of queries and constraints. For the latter problem, an upper bound of of $O\left(m \cdot n^{m}\right)$ can be obtained (c.f. proof of Proposition 6), that is polynomial in the size $n$ of the initial database, but exponential in $m$. So, the problem is tractable in data complexity, but the size of the update sequence is in the exponent of $n$. We are interested in determining if a query can be consistently answered in $O\left(f(m) \times n^{c}\right)$, where $c$ is a constant and $f(m)$ is a function which depends only on $m$, and by doing so, to isolate the complexity introduced through the update.

The area of parameterized complexity (or fixed parameter tractability) [15, $22,30]$ provides the right tools to attack this problem. A decision problem with inputs of the form $(I, p)$, where $p$ is a distinguished parameter of the input, is fixed parameter tractable, and by definition belongs to the class FPT [15], if it can be solved in time $O\left(f(|p|) \cdot|I|^{c}\right)$, where $c$ and the hidden constant do not depend on $|p|$ or $|I|$ and $f$ does not depend on $|I|$.

Definition 7. (parameterized $C Q A$ ) Given a query $Q$, a set of ICs $I C$, and a ground tuple $\bar{t}$, the parameterized complexity of $C Q A$ is the complexity of the decision problem $C Q A^{p}(Q, I C, \bar{t}):=\{(D, U) \mid D$ is an instance, $U$ an update sequence, $\bar{t}$ is consistent answer to $Q$ in $U(D)\}$, whose parameter is $U$, and the consistency of an answer refers to the C-repairs of $U(D)$.

We fixed $Q, I C$ and $\bar{t}$ in the problem definition because, except for the parameter $U$, we are interested in data complexity. We emphasize that we are considering the parameterized version of incremental CQA, and not of CQA in general.

Proposition 7. Incremental CQA for atomic ground queries and functional dependencies under the C-repair semantics is in FPT, being the parameter involved the size $m$ of the update sequence. 
The vertex cover problem belongs to the class FPT, i.e. there is a polynomial time parameterized algorithm that solves it, say $V C(G, k)$, that determines if graph $G$ has a vertex cover of size no bigger than $k[15]$, e.g. there is one that runs in time $O\left(1.2852^{k}+k \cdot n\right)$, being $n$ the size of $G[10]$.

The algorithm whose existence is claimed in Proposition 7 is essentially as follows (c.f. its proof for details): Let $G$ be the conflict graph associated to the database obtained after the insertion of $m$ tuples. By binary search, calling each time $V C\left(G,{ }_{-}\right)$, it is possible to determine the size of a minimum vertex cover for $G$. This gives us the minimum number of tuples that have to be removed in order to restore consistency; and can be done in time $O\left(\log (m) \cdot\left(1.2852^{m}+m \cdot n\right)\right)$, where $n$ is the size of the original database. In order to determine if a tuple $R(\bar{t})$ belongs to every maximum independent set, i.e. if it is consistently true, compute the size of a minimum vertex cover for $G \backslash\{R(\bar{t})\}$. The two numbers are the same iff the answer is yes. The total time is still $\left.O\left(\log (m) \cdot\left(1.2852^{m}+m \cdot n\right)\right)\right)$, which is linear in the size of the original database.

The same algorithm applies if, in addition to tuple insertions, we also have changes of attribute values in the update part; of course, still under the C-repair semantics.

Having established the fixed parameter tractability of CQA, it becomes relevant to find better parameterized algorithms to solve this problem. The proof of Proposition 7 uses the membership to FPT of the vertex cover problem for graphs [15]. That is why we restricted ourselves to functional dependencies, that are associated to conflict graphs. However, the result can be extended to denials constraints. In fact, in this case we have conflict hypergraphs, but the maximum size of an hyperedge is the maximum number of database atoms in a denial constraint, which is determined by the fixed database schema. If this number is $d$, then we are in the presence of the so-called $d$-hitting set problem, consisting in finding the size of a minimum hitting set for an hypergraph with hyperedges bounded in size by $d$. This problem is in FPT [29].

Theorem 2. Incremental CQA for atomic ground queries and denial constraints under the C-repair semantics is in the class FPT, being the parameter involved the size of the update sequence.

The membership to FPT can be extended to the incremental CQA under the possible semantics that sanctions as true what is true of some C-repair. This is due to the reduction of this semantics to the certain semantics exhibited in Section 3, which requires the introduction of only a few extra vertices (this also holds for denial constraints and their hypergraphs).

In a different direction, incremental CQA, considered as a parameterized problem in the size of the update, becomes MONOTONE $W[1]$-hard [15], where the class MONOTONE $W[1]$ is defined as $W[1]$, but in terms of monotone circuits [14]. This result uses a uniform and parameterized reduction from the MONOTONE W[1]-hard problem [14] WEIGHTED MONOTONE 3CNF SAT:

Proposition 8. The parameterized complexity of CQA wrt denial constraints under C-repair semantics is MONOTONE $W[1]$-hard.

Since MONOTONE $W[1]$ coincides with the class FPT [15], we conclude that incremental CQA under this setting is FPT-hard. 


\section{Incremental Complexity of CQA: Other Semantics}

In this section we consider the S-repair semantics based on set difference of tuples, and the A-repair semantics based on changes of attribute values (c.f. Definition 3).

\subsection{S-repair semantics}

Incremental CQA for non-quantified conjunctive queries under denial constraints belongs to PTIME, which can be established by applying the algorithm in [11] for the static case. However, for quantified conjunctive queries the incremental CQA for the S-repair semantics is not in PTIME anymore, which contrast to incremental CQA for the C-repair semantics (c.f. Proposition 6). In fact, by reduction from static CQA for conjunctive queries and denial ICs under the S-repair semantics, which is coNP-hard [11], we obtain

Proposition 9. Under the S-repair semantics, incremental CQA for conjunctive queries and denial constraints is coNP-hard.

Despite the fact that static CQA is harder for the C-repair semantics than for the S-repair semantics for denial constraints $\left(P^{N P(\log (n))}\right.$ vs. coNP-hard), incremental CQA under the S-repair semantics is harder than the same problem under the C-repair semantics. The reason is that for the C-repair semantics the cost of a repair cannot exceed the size of an update, whereas for the S-repair semantics the cost of a repair may be unbounded wrt the size of an update.

Example 4. Consider a schema $R(\cdot), S(\cdot)$ with the denial constraint $\forall x \forall y \neg(R(x) \wedge$ $S(y))$; and the consistent database $D=\{R(1), \ldots, R(n)\}$, with an empty table for $S$. After the update $U=\operatorname{insert}(S(0))$, the database becomes inconsistent, and the S-repairs are $\{R(1), \ldots, R(n)\}$ and $\{S(0)\}$. However, only the former is a C-repair, and is at a distance 1 from the original instance, i.e. as the size of the update. However, the second S-repair is at a distance $n$.

\subsection{A-repair semantics}

Before addressing the problem of incremental complexity, we give a complexity lower bound for the weighted version of static CQA for A-repairs. For this case, we need a weight function $w$ that sends triples of the form $(R(\bar{t}), A$, newValue $)$, where $R(\bar{t})$ is a database tuple stored in the database, $A$ is an attribute of $R$, and newValue is a new value for $A$ in $R(\bar{t})$, to numerical values. The weighted $A$-repair semantics (wA-repair semantics) is just a particular case of Definition $3(\mathrm{c})$, where the distance is given by an aggregation function $g$ applied to the set of numbers $\{w(R(\bar{t}), A$, newValue $) \mid R(\bar{t}) \in D\}$.

Typically, $g$ is the sum, and the weights are $w(R(\bar{t}), A$, newValue $)=1$ if $R(\bar{t})[A]$ is different from newValue, and 0 otherwise, where $R(\bar{t})[A]$ is the projection of database tuple $R(\bar{t})$ on attribute $A$. That is, just the number of changes is counted. However, in [6], $g$ is still the sum, but the weight function is given by $w(R(\bar{t}), A$, newValue $)=\alpha_{A} \cdot(R(\bar{t})[A]-\text { newValue })^{2}$, where $\alpha_{A}$ is a coefficient introduced to capture the relative importance of attribute $A$ or scale factors. 
Theorem 3. Static CQA for ground atomic queries and denial constraints under the wA-repair semantics is $P^{N P}$-hard.

To obtain complexity lower bounds for incremental CQA under this repair semantics, we need first a technical result

Lemma 4. For any planar graph $G$ with vertices of degree at most 4 , there exists a regular graph $G^{\prime}$ of degree 4 that is 4 -colorable, such that $G^{\prime}$ is 3colorable iff $G$ is 3-colorable, and $G^{\prime}$ can be built in polynomial time in the size of $G$.

Notice that graph $G$, due to its planarity, is 4-colorable. The graph $G^{\prime}$, is an extension of graph $G$ that may not be planar, but preserves 4-Colorability. Now, from Lemma 4 and the $N P$-hardness of 3-colorability for planar graphs with vertices of degree at most 4 [20, theorem 2.3], we obtain

Corollary 3. 3-Colorability for regular graphs of degree 4 (i.e. with all their vertices of exactly degree 4 ) is $N P$-complete.

We use the construction in Lemma 4 as follows: Given any planar graph $G$ of degree 4 , we construct graph $G^{\prime}$ as in the lemma, which is regular of degree 4 and 4-colorable. Its 4-colorability is encoded as a database problem with a fixed set of first-order constraints. Since $G^{\prime}$ is 4-colorable, the database is consistent. Furthermore, $G^{\prime}$ uses all the 4 colors available in the official table of colors, as specified by the ICs. In the update part, deleting one of the official colors leaves us with the problem of coloring graph $G^{\prime}$ with only the three remaining colors (under an A-repair semantics only changes of colors are allowed to restore consistency), which is possible iff the original graph $G$ is 3-colorable. Deciding about the latter problem is $N P$-complete [20]. We obtain

Theorem 4. For A-repairs, ground atomic queries, first-order ICs, and update sequences consisting of tuple deletions, incremental CQA is coNP-hard.

This result applies to first-order ICs and delete operations. For incremental CQA in general, update operations that introduce violations can be in principle of any of the forms insert, delete, change. Of course, the hardness result just obtained then trivially applies to general update sequences.

In order to obtain a hardness result for denial constraints (for which we are assuming update sequences do not contain tuple deletions), we can use the kind of A-repairs introduced in [6].

Theorem 5. Incremental CQA wrt denial constraints and atomic queries under the wA-repair semantics is $P^{N P}$-hard.

Under the attribute-based repairs semantics, if the update sequence consist of change actions, then we can obtain polynomial time incremental CQA under the additional condition that the set of attribute values than can be used to restore consistency is bounded in size, independent from the database (or its active domain). Such an assumption can be justified in several applications, like in census-like databases that are corrected according to inequality-free denial constraints that force the new values to be taken in the border of a database 
independent region [6]; and also in applications where denial constraints, this time containing inequalities, force the attribute values to be taken in a finite, prespecified set. The proof is similar to the one of Proposition 6, and the polynomial bound now also depends on the size of the set of candidate values.

Theorem 6. Under A-repairs that can be obtained using values from a databaseindependent bounded set, incremental CQA for first-order boolean queries, denial constraints, and update sequences containing only change actions is in PTIME in the size of the original database.

\section{Conclusions}

The dynamic scenario for consistent query answering that considers possible updates on a database had not been considered before in the literature. Doing incremental CQA on the basis of the original database and the sequence of updates is an important and natural problem. Developing algorithms that take into account previously obtained consistent answers that are possible cached and the updates at hand is a crucial problem for making CQA scale up for real database applications. Much research is still needed in this direction.

In this paper we have concentrated mostly on complexity bounds for this problem under different semantics. When we started obtaining results for incremental CQA under repairs that differ from the original instance by a minimum number of tuples, i.e. C-repairs, we realized that this semantics had not been sufficiently explored in the literature in the static version of CQA, and that a comparison was not possible. In the first part of this paper we studied the complexity of CQA for this semantics. In doing so, we have developed graph theoretic techniques that allow us to connect the certain and possible (or cautious and brave) semantics for CQA.

Our results show that the incremental complexity is lower than the static one in several useful cases, but sometimes the complexity cannot be lowered. It is a subject of ongoing work the development of concrete and explicit algorithms for incremental CQA. Also the complexity of incremental CQA under the alternative semantics presented in Section 5 deserves further investigation and a more complete picture still has to emerge.

We obtained the first results about fixed parameter tractability for incremental CQA, where the input, for a fixed database schema, can be seen as formed by the original database and the update sequence, whose length is a relevant parameter. This problem requires additional investigation. It would be interesting to examine the area of CQA in general from the point of view of parameterized complexity, and not only the incremental case. For example, other natural candidates to be a parameter in the classic, static setting could be: (a) the number of inconsistencies in the database, (b) the degree of inconsistency, i.e. the maximum number of violations per database tuple, (c) complexity of inconsistency, i.e. the length of the longest path in the conflict graph or hypergraph. These parameters may be practically significant since in many applications, like census application [6], inconsistencies are "local".

We considered a version of incremental CQA that assumes that the database is already consistent before updates are executed, a situation that could have 
been achieved because no previous updates violated the given semantic constraints or a repaired version was chosen before the new updates were executed.

We are currently investigating the dynamic case of CQA in the frameworks of dynamic complexity [23, 33] or incremental complexity as introduced in [27]. In this case we start with a database $D$ that is not necessarily consistent -and this is the main new issue involved- on which a sequence of basic update operations $U_{1}, U_{2}, \ldots, U_{m}$ is executed. A clever algorithm for CQA may create or update intermediate data structures at each atomic update step, to help obtain answers at subsequent steps. We are interested in the computational complexity of CQA after a sequence of updates, when the data structures created by the query answering algorithm at previous states are themselves updatable and accessible.

Acknowledgments: Research supported by NSERC, and EU projects: Knowledge Web, Interop and Tones. L. Bertossi is Faculty Fellow of IBM Center for Advanced Studies (Toronto Lab.). Part of this research was done while L. Bertossi visited the University of Bolzano during the summer 2005; he appreciates the hospitality and support of Enrico Franconi and the KRDB group.

\section{References}

[1] Abiteboul, S., Hull, R. and Vianu, V. Foundations of Databases. Addison-Wesley, 1995.

[2] Arenas, M., Bertossi, L. and Chomicki, J. Consistent Query Answers in Inconsistent Databases. Proc. ACM Symposium on Principles of Database Systems (PODS 99), 1999.

[3] Arenas, M., Bertossi, L. and Chomicki, J. Answer Sets for Consistent Query Answering in Inconsistent Databases. Theory and Practice of Logic Programming, 2003, 3(4-5):393-424.

[4] Arenas, M., Bertossi, L., Chomicki, J., He, X., Raghavan, V. and Spinrad, J. Scalar Aggregation in Inconsistent Databases. Theoretical Computer Science, 2003, 296:405-434.

[5] Bertossi, L. and Bravo, L. Consistent Query Answers in Virtual Data Integration Systems. In Inconsistency Tolerance, Springer LNCS 3300, 2004, pp. 42-83.

[6] Bertossi, L., Bravo, L., Franconi, E. and Lopatenko, A. Fixing Numerical Attributes under Integrity Constraints. Proc. Tenth International Symposium on Database Programming Languages (DBPL 05), Springer LNCS 3774, 2005, pp. 262-278.

[7] Bertossi, L. and Chomicki, J. Query Answering in Inconsistent Databases. In Logics for Emerging Applications of Databases. Springer, 2003, pp. 43-83.

[8] Buccafurri, F., Leone, N. and Rullo, P. Enhancing Disjunctive Datalog by Constraints. IEEE Transactions on Knowledge and Data Engineering, 2000, $12(5): 845-860$.

[9] Cali, A., Lembo, D. and Rosati, R. Complexity of Query Answering over Inconsistent and Incomplete Databases. Proc. ACM Symposium on Principles of Database Systems (PODS 03), 2003, pp. 260-271.

[10] Chen, J., Kanj, I. and Jia, W. Vertex Cover: Further Observations and Further Improvements. In Proc. 25th International Workshop on Graph-Theoretic Concepts in Computer Science (WG 99), Springer LNCS 1665, 1999, pp. 313-324.

[11] Chomicki, J. and Marcinkowski, J. Minimal-Change Integrity Maintenance using Tuple Deletions. Information and Computation, 2005, 197(1-2):90-121. 
[12] Chou, T. and Winslett, M. A Model-Based Belief Revision System. J. Automated Reasoning, 1994, 12:157-208.

[13] Dalal, M. Investigations into a Theory of Knowledge Base Revision: preliminary report. Proc. Seventh National Conference on Artificial Intelligence (AAAI 88), 1988, pp. 475-479.

[14] Downey, R.G. and Fellows, M.R. Fixed-Parameter Tractability And Completeness I: Basic Results. SIAM J. Computing, 1995, 24(4):873-921.

[15] Downey, R.G. and Fellows, M.R. Parameterized Complexity. Springer, Monographs in Computer Science, 1999.

[16] Eiter, T. and Gottlob, G. On the Complexity of Propositional Knowledge Base Revision, Updates, and Counterfactuals. Artificial Intelligence, 1992, 57(2-3):227270.

[17] Flesca, S., Furfaro, F. Parisi, F. Consistent Query Answers on Numerical Databases under Aggregate Constraints. Proc. Tenth International Symposium on Database Programming Languages (DBPL 05), Springer LNCS 3774, 2005, pp. 279-294.

[18] Franconi, E., Laureti Palma, A., Leone, N., Perri, S. and Scarcello, F. Census Data Repair: a Challenging Application of Disjunctive Logic Programming. In Proc. Logic for Programming, Artificial Intelligence, and Reasoning (LPAR 01), Springer LNCS 2250, 2001, pp. 561-578.

[19] Fuxman, A. and Miller, R. First-Order Query Rewriting for Inconsistent Databases. Proc. International Conference on Database Theory (ICDT 05), Springer LNCS 3363, 2004, pp. 337-351.

[20] Garey, M., Johnson, D. and Stockmeyer, L. Some Simplified NP-Complete Graph Problems. Theoretical Computer Science, 1976, 1(3):237-267.

[21] Greco, G. and Lembo, D. Data Integration with Preferences Among Sources. Proc. International Conference on Conceptual Modeling (ER 04), Springer LNCS 3288, 2004, pp. 231-244.

[22] Grohe, M. Parameterized Complexity for the Data-base Theorist. SIGMOD Record, 2002, 31(4):86-96.

[23] Immerman, N. Descriptive Complexity. Graduate Texts in Computer Science. Springer, 1999.

[24] Krentel, M. The Complexity of Optimization Problems. J. Computer and Systems Sciences, 1988, 36:490-509.

[25] Leone, N., Pfeifer, G., Faber, W., Eiter, T., Gottlob, G., Perri, S. and Scarcello, F. The DLV System for Knowledge Representation and Reasoning. To appear in ACM Transactions on Computational Logic.

[26] Libkin, L. and Wong, L. On the Power of Incremental Evaluation in SQL-Like Languages. Proc. International Workshop on Database Programming Languages (DBPL 99),Springer LNCS 1949, 1999, pp. 17-30.

[27] Miltersen, P.B., Subramanian, S., Vitter, J.S. and Tamassia, R. Complexity Models for Incremental Computation. Theoretical Computer Science, 1994, 130(1):203236.

[28] Nicolas, J-M. Logic for Improving Integrity Checking in Relational Data Bases. Acta Informatica, 1982, 18:227-253.

[29] Niedermeier, R. and Rossmanith, P. An Efficient Fixed-Parameter Algorithm for 3-Hitting Set. Journal of Discrete Algorithms, 2003, 1(1):89-102.

[30] Papadimitriou, C.H. and Yannakakis, M. On the Complexity of Database Queries. J. Comput. Syst. Sci., 1999, 58(3):407-427.

[31] Papadimitriou, C. Computational Complexity. Addison-Wesley, 1994.

[32] Robertson, N., Sanders, D.P., Seymour, P. and Thomas, R. Eficiently FourColoring Planar Graphs. In Proc. 28th ACM Symposium on the Theory of Computing (STOC 96), ACM Press, 1996, pp. 571-575. 
[33] Weber, V. and Schwentick, T. Dynamic Complexity Theory Revisited. Proc. Annual Symposium on Theoretical Aspects of Computer Science (STACS 05), Springer LNCS 3404, 2005, pp. 256-268.

[34] Widom, J. and Ceri, S. Active Database Systems: Triggers and Rules For Advanced Database Processing. Morgan Kaufmann, 1996.

[35] Wijsen, J. Condensed Representation of Database Repairs for Consistent Query Answering. Proc. International Conference on Database Theory (ICDT 03), Springer LNCS 2572, 2003, pp. 378-393.

\section{Appendix: Proofs}

Proof of Lemma 1: We consider the three cases for membership of $v$ to maximum independent sets in $G$. Let $m$ be the cardinality of a maximum independent set in $G$. We establish now the first bi-conditional. The second bi-conditional follows directly from the analysis for the first one.

(a) Assume that $v$ belongs to a maximum independent set $I$ of $G$. In this case, $v^{\prime}$ can be added to $I$ obtaining an independent set of $G^{\prime}$. In this case $\left|I \cup\left\{v^{\prime}\right\}\right| \geq m+1$.

Assume that $v$ does not belong a some maximum independent set $I^{\prime}$ of $G^{\prime}$. If $v \notin I^{\prime}$, then some of its neighbors belong to $I^{\prime}$, and then, $v^{\prime} \notin I^{\prime}$. In consequence, $I^{\prime}$ is also a maximum independent set of $G$. Then, $\left|I^{\prime}\right|=m$. But this is not possible, because the size of independent set of $I^{\prime}$ is at least $m+1$.

(b) Assume that $v$ does not belong to any maximum independent sets of $G$. Then, some of it neighbors can be found in every maximum independent set of $G$, and none of them can be extended with $v^{\prime}$ to become an independent set of $G^{\prime}$.

So, all the maximum independent set of $G$ are maximum independent sets of $G^{\prime}$ of size $m$.

Assume, that $v$ belongs to all maximum independent sets of $G^{\prime}$. Then none of the neighbors of $v$ can be found in independent sets of $G$, and then $v^{\prime}$ can be found in all the maximum independent sets of $G^{\prime}$. Since the maximum independent sets of $G^{\prime}$ have at least cardinality $m$, it must hold that the maximum independent sets of $G^{\prime}$ have cardinality at least $m+1$. Then the deleting $v^{\prime}$ from all the maximum independent sets of $G^{\prime}$ will give us independent sets of $G$ of size at least $m$, i.e. maximum independent sets of $G$. To all of them $v$ belongs. A contradiction.

Proof of Lemma 2: (sketch) Hang a rhombus from $v$, i.e. add three other vertices, two of them connected to $v$, and the third one, connected to the two previous ones. Then, reason by cases as in the proof of Lemma 1.

Proof of Proposition 1: For the first claim, given a graph $G$ and a vertex $v$, build in polynomial time the graph $G^{\prime}$ as in Lemma 1. It holds that $v$ belongs to some maximum independent set of $G$ iff $v$ belongs to every maximum independent set of $G^{\prime}$. Now, $v$ belongs to every maximum independent set of $G^{\prime}$ iff $\mid$ maximum independent set in $G^{\prime}|-|$ maximum independent set in $G \mid=1$.

Since computing the maximum cardinality of a clique can be done in time $F P^{N P(\log (n))}[24]$ (see also [31, theorem 17.6]), computing the maximum cardinality of an independent set can be done in the same time (just consider the 
complement graph). In consequence, in order to decide about $v$ and $G$, we can compute the cardinalities of the maximum independent set for $G$ and $G^{\prime}$ in 2 times $F P^{N P(\log (n))}$, and next compute their difference. It total, we can perform the whole computation in $F P^{N P(\log (n))}$. In consequence, by definition of class $F P^{N P(\log (n))}$, we can decide by means of a polynomial time machine that makes $O(\log (n))$ calls to an $N P$ oracle, i.e. the decision is made in time $P^{N P(\log (n))}$. The same proof works for the second claim. It can also be obtained from the first claim and Lemma 2.

Proof of Corollary 1: Construct the conflict graph for the instance wrt the FDs. An atomic ground query is consistently true if the corresponding vertex in the conflict graph belongs to all the maximum independent sets. Then use Proposition 1.

Proof of Proposition 2: Consider a graph $G=\langle V, E\rangle$, and assume the vertices of $G$ are uniquely labelled. Consider the database schema with two relations, $\operatorname{Vertex}(v)$ and $\operatorname{Edges}\left(v_{1}, v_{2}, e\right)$, and the denial constraint $\forall v_{1} v_{2} \neg\left(\operatorname{Vertex}\left(v_{1}\right) \wedge\right.$ $\left.\operatorname{Vertex}\left(v_{2}\right) \wedge \operatorname{Edges}\left(v_{1}, v_{2}, e\right)\right)$. Vertex stores the vertices of $G$. For each edge $\left\{v_{1}, v_{2}\right\}$ in $G$, Edges contains $n$ tuples of the form $\left(v_{1}, v_{2}, i\right)$, where $n$ is the number of vertices in $G$. All the values in the third attribute of Edges are different, say from 1 to $n|E|$. The size of the database instance obtained trough this padding of $G$ is still polynomial in size.

This instance is highly inconsistent, and its C-repairs are all obtained by deleting vertices, i.e. elements of Vertex alone. In fact, an instance such that all tuples but one in Vertex are deleted, but all tuples in Edges are preserved is a consistent instance. In this case, $n-1$ tuples are deleted. If we try to achieve a repair by deleting tuples from Edges, say $\left(v_{1}, v_{2}, i\right)$, then in every repair of that kind all the $n$ tuples of the form $\left(v_{1}, v_{2}, j\right)$ have to be deleted as well. This would not be a minimal cardinality repair.

Assume that $I$ is a maximum cardinality independent set of $G$. The deletion of all tuples $(v)$ from Vertex, where $v$ does not belong to $I$, is a C-repair. Now, assume that $D$ is a repair. As we know, only tuples from Vertex may be deleted. Since, in order to satisfy the constraint, no two vertices in the graph that belong to $D$ are adjacent, the vertices remaining in Vertex form an independent set in $G$.

In general, the number of deleted tuples is equal to $n-|I|$, where $I$ is an independent set represented by a repair. So each minimal cardinality repair corresponds to a maximum independent set and vice-versa.

Proof of Corollary 2: This follows from Proposition 2, the fact that C-repairs correspond to maximum cliques in the complement of the conflict graph [4], and the $P^{N P(\log (n))}$-completeness of determining the size of a maximum clique [24].

Proof of Proposition 3: We use the conflict hypergraph. The problem of determining the maximum clique size for hypergraphs is in $F P^{N P(\log (n)}$ by the same argument as for conflict graphs: Deciding if the size of maximum clique is greater than $k$ is in $N P$. So, by asking a logarithmic number of $N P$ queries, we can determine the size of maximum clique. 
The membership to $P^{N P(\log (n))}$ of CQA for the C-semantics still holds for conjunctive queries without existential variables. In fact, given an inconsistent database $D$, a query $Q$, and a ground tuple $t$, we check if $t$ is consistent answer to $Q$ from $D$ as follows: Check if $t$ is an ordinary answer to $Q$ in $D$ (without considering the constraints). If not, the answer is no.

Otherwise, let $t_{1}, \ldots, t_{k}$ be the database tuples which are answers to $Q$ in $D$ and produce $t$ as an answer. Since $Q$ does not contain existential variables, only one such set exists. Compute the size of a maximum independent set for the graph representation of $D$, say $m_{0}$. Compute the size of a maximum independent set for the graph representation of $D \backslash\left\{t_{1}\right\}$, say $m_{1}$. If $m_{1}=m_{0}$, then there exist a maximum independent set of $D$ that does not contain $t_{1}$. So, there exists a minimum repair that does not satisfy that $t$ is an answer to $Q$. If $m_{1}<m_{0}$, repeat this procedure for all tuples in $t_{1}, \ldots, t_{k}$. Thus, we have to pose $k$ queries (that is determined only by the size of the query) to an $F P^{N P(\log (n))}$ oracle.

In consequence, the complexity of CQA for conjunctive queries without existential variables is in $P^{N P(\log (n))}$.

Proof of Lemma 3: The new graph $G^{\prime}$ consists of two copies of $G$, say $G_{1}, G_{2}$, two additional graphs, $I_{k}, I_{k+1}$, and two extra vertices $t, b$. Subgraph $I_{k}$ consists of $k$ mutually disconnected vertices; subgraph $I_{k+1}$ consists of $k+1$ mutually disconnected connected vertexes. Each vertex of $G_{1}$ is adjacent to each vertex of $I_{k}$, and each vertex of $G_{2}$ is adjacent to each vertex of $I_{k+1}$. Each vertex of $I_{k}$ is adjacent to $t$, and each vertex of $I_{k+1}$ is adjacent to $b$. Finally, $t, b$ are connected by an edge (c.f. Figure 1).

We claim that vertex $t$ belongs to all maximum independent sets of $G^{\prime}$ iff the cardinality of maximum independent set of $G$ is equal to $k$. To prove this claim, we consider a few, but representative possible cases. With $I(G)$ we denote an arbitrary maximum independent set of $G$.

1. $|I(G)|<k-1$ : The maximum independent set of $G^{\prime}$ is $I_{k} \cup I_{k+1}$; with cardinality $2 k+1$.

2. $|I(G)|=k-1$ : The maximum independent sets of $G^{\prime}$ are (a) $I\left(G_{1}\right) \cup I_{k+1} \cup$ $\{t\}$, and (b) $I_{k} \cup I_{k+1}$, with cardinality $2 k+1$.

3. $|I(G)|=k$ : The maximum independent set of $G^{\prime}$ is $I_{k+1} \cup I\left(G_{1}\right) \cup\{t\}$, with cardinality $2 k+2$.

4. $|I(G)|=k+1$ : The maximum independent sets of $G^{\prime}$ are (a) $G_{1} \cup G_{2} \cup\{t\}$, (b) $G_{1} \cup G_{2} \cup\{b\}$, (c) $G_{1} \cup I_{k+1} \cup\{t\}$; with cardinality $2 k+3$.

5. $|I(G)|>k+1$ : The maximum independent sets of $G^{\prime}$ are (a) $G_{1} \cup G_{2} \cup\{t\}$, (b) $G_{1} \cup G_{2} \cup\{b\}$; with cardinality $2|I|+1$.

Only in case $|I(G)|=k, t$ belongs to all maximum independent sets.

Proof of Proposition 4: By reduction from the following $P^{N P(\log (n))}$-complete decision problem [24, theorem 3.5]: Given a graph $G$ and an integer $k$, is the size of a maximum clique in $G$ equivalent to $0 \bmod k$ ?

Assume graph $G$ has $n$ vertices. We can also assume that $k$ is not bigger than $n$. Now, we pass to the graph $G^{\prime}$ that is the complement of $G$ : It has the same vertices as $G$, with every two distinct vertices being adjacent in $G^{\prime}$ iff they are not adjacent in $G$. A maximum independent set of $G^{\prime}$ is a maximum clique of 
$G$ and vice-versa. So, the cardinality of a maximum independent set of $G^{\prime}$ is the size of a maximum clique of $G$.

Next, we take advantage of the construction in Lemma 3 (c.f. Figure 1): For each $m \in\left\{k, 2 k, \cdots,\left\lfloor\frac{n}{k} \times k\right\rfloor\right\}$, construct the block graph $B_{m}\left(G^{\prime}, t_{m}\right)$. (There are $[n / k]$ possible solutions to the equation $x \equiv 0 \bmod k$.) All these graphs are disconnected from each other. Next, create a new vertex $t_{g}$ and connect it to the vertices $t_{m}$ of the blocks $B_{m}\left(G^{\prime}, t_{m}\right)$. It is easy to check that the resulting graph, say $\bar{G}$, has its size bounded above by $O\left(n^{4}\right)$.

It holds that vertex $t_{g}$ does not belong to every maximum independent set of $\bar{G}$ iff the size of maximum independent set of $G$ is equivalent to $0 \bmod k$. So, we have a reduction to the complement of our problem, but the class $P^{N P(\log (n))}$ is closed under complement.

In fact, if the size of maximum independent set of $G$ is not equivalent to $0 \bmod k$, then for every block $B$ in $\bar{G}$, there exists a maximum independent set $I_{B}$ of the block $B$ such that $t_{B} \notin I_{B}\left(t_{B}\right.$ is the top node of block $\left.B\right)$. The maximum independent set of $\bar{G}$ is $\left\{t_{g}\right\} \cup \bigcup_{B} I_{B}$ (because there are no edges between blocks and between $t_{g}$ and other vertices besides $t_{B}$ ). Consider any independent set $I$ of $\bar{G}$ that does not contain $t_{g}$. The size of the projection of $I$ on any block is not greater than the size of the maximum independent set of the block; so $|I| \leq\left|\bigcup_{B} I_{B}\right|$. So, $t_{g}$ belongs to every maximum independent set of $\bar{G}$.

Now, if the size of a maximum independent set of $G$ is equivalent to $0 \bmod k$, then there exists one block $B_{o}$ such that $t_{B_{o}}$ belongs to every maximum independent set $I_{B_{o}}$ of $B_{o}$, while for all other blocks $B$ there exists $I_{B}$ such that $t_{B} \notin I_{B}$. Consider a maximum independent set $I_{t}$ of $\bar{G}$ that contains $t_{G}$.

Every maximum independent set of $\bar{G}$ that contains $t_{g}$ is of the form $\left\{t_{g}\right\}$ union of maximum independent sets from the blocks $B$ other than $B_{o}$ that do not contain their corresponding $t_{B}$ union any maximum independent set of $B_{o} \backslash\left\{t_{B_{o}}\right\}$. The size of such a set is $s=1+\sum_{B \neq B_{o}}|I(B)|+\left(\left|I_{B_{o}}\right|-1\right)$. A maximum independent set $I$ that does not contain $t_{g}$, is the union of maximum independent sets $I_{B}$ of all the blocks $B$ of $\bar{G}$, and its size is equal to $\sum_{B}\left|I_{B}\right|$, i.e. $s$. Then, there exists a maximum independent set that does not contain $t_{g}$.

Proof of Theorem 1: Membership follows from Proposition 3. Now we prove hardness. For a graph $G$ and integer $k$, we construct a database $D$, such that the consistent answer to a ground atomic query $Q$ can be used to decide if the size of a maximum clique of $G$ is equivalent to $0 \bmod k$ (c.f. proof of Proposition 4). Construct the graph $\bar{G}$ as in Proposition 4. Encode graph $\bar{G}$ as a database inconsistency problem, introducing a unary relation $V$ (for vertices) and $E$ (3-ary), where $E$ corresponds to the edge relation in $\bar{G}$ plus a third padding attribute to make changing it more costly. For each vertex $v \in \bar{G}$, there is a tuple $(v)$ in $V$.

We also introduce the denial constraint: $\forall v_{1} \forall v_{2} \neg\left(V\left(v_{1}\right) \wedge V\left(v_{2}\right) \wedge E\left(v_{1}, v_{2},-\right)\right)$ (an underscore means any variable implicitly universally quantified). For each edge $\left\{v_{1}, v_{2}\right\} \in \bar{G}$, create $n$ different versions $\left(v_{1}, v_{2}, p\right)$ in $E$, as in the proof of Proposition 2. The effect of fixing the database wrt the given denial constraint may be the removal of tuples representing vertices or/and the removal of tuples representing edges. We want to forbid the latter alternative because those repairs do not represent maximum independent set; and this is achieved by making them more expensive than vertex removal through the padding process. 
The consistent answer to the query $V\left(t_{g}\right)$ is no, i.e. not true in all repairs, iff $t_{g}$ does not belong to all maximum independent sets of $\bar{G}$ iff the size of a maximum independent set of $G^{\prime}$ is equivalent to $0 \bmod k$ iff the size of a maximum clique of $G$ is equivalent to $0 \bmod k$.

Proof of Proposition 5: Membership is proved as for the C-repair semantics (c.f. Proposition 3): Repairs correspond to maximum weighted independent sets of the associated hypergraph $G$. The weight of a maximum weighted independent set can be found in $P^{N P}$ (as for independent set, but $\log \left(O\left(2^{n}\right)\right)=\operatorname{poly}(n)$ oracle calls are required). To check if a vertex $v$ belongs to all maximum weighted independent sets, it is good enough to compute weights of maximum independent sets for $G$ and $G \backslash\{v\}$.

Proof of Proposition 6: For denial constraints tuple deletions do not introduce any violations, so we consider a sequence $U$ consisting of tuple insertion and updates.

Assume that $k$ of the $m$ inserted tuples violate ICs, perhaps together with some tuples already in $D$. If we delete $k$ violating tuples, then we get a consistent database $D^{\prime}$; so a minimal repair is at a distance less than or equal to $k$ from $D$. To find all minimal repairs it is good enough to check no more than $N=\left(\begin{array}{c}n+m \\ 1\end{array}\right)+\left(\begin{array}{c}n+m \\ 2\end{array}\right)+\cdots+\left(\begin{array}{c}n+m \\ k\end{array}\right)$ repairs, where $|D|=n$. If $m$ is small, say less than $c \cdot n$, then $N<k\left(\begin{array}{c}n+m \\ k\end{array}\right) \leq m\left(\begin{array}{c}n \\ m\end{array}\right)^{m}<m n^{m}$. Thus, the incremental complexity of the CQA is polynomial wrt $n$.

In case $U$ contains change updates, the proof is essentially the same, but the role of $m$ is taken by $m \cdot a$, where $a$ is the maximum arity of the relations involved. This is because we have to consider possible changes in different attributes.

Proof of Proposition 7: First, it is known that the problem of, given a graph $G$ and a number $k$, determining if there exists a vertex cover of size less than or equal to $k$ is in FPT [15]. We will use this problem to solve ours.

Now, let us assume that we have a consistent database $D$ of size $n$, and we update it inserting $k$ new tuples, obtaining an inconsistent database $D^{\prime}$ with conflict graph $G$. The size of $G$ is $O(n)$ by our assumption on the size of $m$ in comparison with $n$. Every C-repair of $D^{\prime}$ is a maximum independent set of $G$, and can be obtained by deleting from $G$ a minimum vertex cover, because the problems are complementary. So, a minimum vertex cover corresponds to the vertices that are to be deleted to obtain a repair.

Since the original database $D$ is consistent, the vertices of $G$ corresponding to database tuples in $D$ are all disconnected from each other. In consequence, edges may appear only by the update sequence, namely between the $m$ new tuples or between them and the elements of $D$. Then, we know that there is a vertex cover for $G$ of size $m$. However, we do not know if it is minimum.

In order to find the size of a minimum vertex cover of $G$, we may start doing binary search from $m$, applying an FPT algorithm for vertex cover. Each check for vertex cover, say for value $m_{i}$, can be done in $O\left(1.2852_{i}^{m}+m_{i} \cdot n\right)$ [10]. Then $\log (m)$ checks take time $O\left(\log (m) \cdot\left(1.2852^{m}+m \cdot n\right)\right) \leq O(f(m) \cdot n)$, with $f$ an exponential function in $m$. So, it is in FPT obtaining the size of a minimum 
vertex cover for $G$, which gives us the minimum number of tuples to remove to restore consistency.

Now, for CQA we want to check if a vertex $R(\bar{t})$ belongs to all maximum independent sets of $G$, which happens if it does not belong to any minimum vertex covers. This can be determined by checking the size of minimum vertex cover for $G^{\prime}$ and $G^{\prime} \backslash\{R(\bar{t})\}$. If they are the same, then $R(\bar{t})$ belongs to all maximum independent sets and the consistent answer to the query $R(\bar{t})$ is yes.

Proof of Proposition 8: By uniform reduction from the MONOTONE W[1]hard problem [14] WEIGHTED MONOTONE 3CNF SAT, which is defined as follows: Given a $3 \mathrm{CNF}$ monotone circuit $C$ and an integer $k$, is it possible to make exactly $k$ of the inputs 1 and obtain output 1 for $C$ ?

The database schema consists of relations $\operatorname{Clause}\left(C, V_{1}, V_{2}, V_{3}, p\right), \operatorname{Var}(V)$, $\operatorname{Cond}(X, Y)$, where $p$ and $Y$ are dummy variables intended to create many copies of a tuple, to forbid the deletion of those tuples by making the potential repair too costly. The integrity constraint is $\forall C V_{1} V_{2} V_{3} p y \neg\left(\operatorname{Clause}\left(C, V_{1}, V_{2}, V_{3}, p\right) \wedge\right.$ $\left.\operatorname{Var}\left(V_{1}\right) \wedge \operatorname{Var}\left(V_{2}\right) \wedge \operatorname{Var}\left(V_{3}\right) \wedge \operatorname{Cond}(1, y)\right)$. Given a monotone $3 \mathrm{CNF}$ formula $\Psi=\psi_{1} \wedge \psi_{2} \wedge \cdots \wedge \psi_{m}$ and a parameter $k$, for each clause $\psi_{i}=\left(x_{i_{1}} \vee x_{i_{2}} \vee x_{i_{3}}\right)$, where the $x_{i_{j}}$ are atoms, store in Clause $n$ copies of the form $\left(i, x_{i_{1}}, x_{i_{2}}, x_{i_{3}}, p\right)$ (replace variable by any new constant if a clause has less than three variables). For each variable $x$ in $\Psi$, store $x$ in $\operatorname{Var}$. $C$ is initially empty. The resulting database is consistent.

Now, on the update part, insert $(1, i)$ into Cond $, i=1, \ldots k$. Then there exists an assignment with weight less than $k$ iff $\operatorname{Cond}(1,1)$ is false in every repair.

Since we have to determine if there exists a satisfying assignment with weight exactly $k$, it is good enough to ask a query to two databases, built as before, but for both $k$ and $k+1$, which is compatible with the definition of parametric reduction, that allows to use of a constant number of instances. In our case, since we have that: (a) if weight $<k$, then consistent answer is yes, (b) if weight is equal to $k$, then the consistent answer is false (i.e. false in all repairs), and (c) if weight $>k$, the consistent answer is false. So, we construct two instances, for $k$ and $k+1$. The weight is equal to $k$ iff the consistent answer for the first instance is false and for the second one it is yes.

Proof of Proposition 9: By reduction from static CQA for (existentially quantified) conjunctive queries and denial ICs under minimal set semantics, which is coNP-hard [11]. Consider an instance for this problem consisting of a database $D$, a set of denial ICs $I C$, and a query $Q$.

For every denial $i c \in I C$, pick up a relation $R^{i c}$ in it and expand it to a relation $\overline{R^{i c}}$ with an extra attribute Control. Also add a new, one attribute relation Controler $(A)$. Next, transform each integrity constraint ic: $\forall \bar{x} \neg(P(\bar{x}) \wedge \cdots \wedge$ $\left.R^{i c}(\bar{x}) \wedge \cdots \wedge \gamma\right)$ into $i c^{\prime}: \forall \bar{x} \forall$ contr $\neg\left(P(\bar{x}) \wedge \cdots \wedge \overline{R^{i c}}(\bar{x}\right.$, contr $) \wedge$ Controler $($ contr $) \wedge$ $\gamma)$. We obtain a set $I C^{\prime}$ of denial constraints. The original database $D$ is extended to a database $\bar{D}$ with the new relation Controler, which is initially empty, and the relations $\overline{R^{i c}}$, whose extra attributes Contr initially take all the value 1 . Due to the extension of Controler, $I C^{\prime}$ is satisfied.

Now in the incremental context, we consider the inconsistent instance $\bar{D}^{\prime}$ obtained via the update insert (Controler (1)) on $\bar{D}$. The S-repairs of $\bar{D}^{\prime}$ wrt $I C^{\prime}$ 
are: (a) $\bar{D}$ and (b) all the S-repairs of $\bar{D}$ (plus the tuple Controler(1) in each of them), which are in one-to-one correspondence with the S-repairs of $D$ wrt $I C$. Now, for a conjunctive query $Q$ in the language of $D$, produce the conjunctive query $Q^{\prime}: \exists \cdots y_{i c} \cdots Q \frac{\cdots R^{i c}(\bar{x}) \cdots}{\cdots \overline{R^{i c}}\left(\bar{x}, y_{i c}\right) \cdots}$ in the language of $\bar{D},{ }^{3}$ where each atom $\left.R^{i c}(\bar{x})\right)$ in $Q$ is replaced by $\exists y_{i c} \overline{R^{i c}}\left(\bar{x}, y_{i c}\right)$.

Notice that all the repairs in (b) are essentially contained in $\bar{D}$, except for the tuple Controler(1), whose predicate does not appear in the queries. This is because denial constraints are obtained by tuple deletions. In consequence, any answer to the conjunctive (and then monotone) query in a repair in (b) is also an answer in the repair in (a). In consequence, the repair $\bar{D}$ does not contribute with any new consistent answers, neither invalidates any answers obtained by the repairs in (b). So, it holds $C q a(Q, D, I C)=C q a\left(Q^{\prime}, \bar{D}^{\prime}, I C^{\prime}\right)$.

Proof of Theorem 3: We can adapt the proof of theorem 4 in [6] about the $\Delta_{2}^{P}$ hardness of CQA under minimum square distance. We provide a $L O G S P A C E$ reduction from the following problem [24, theorem 3.4]: Given a Boolean formula $\psi\left(X_{1}, \cdots, X_{n}\right)$ in $3 \mathrm{CNF}$, decide if the last variable $X_{n}$ is equal to 1 in the lexicographically maximum satisfying assignment (the answer is $N o$ if $\psi$ is not satisfiable).

Create a database schema with relations: Clause(id, $\operatorname{Var}_{1}, V l_{1}, V r_{2}, V l_{2}$, $\left.\operatorname{Var}_{3}, \operatorname{Val}_{3}\right)$, Var (var, val), Dummy $(x)$, with denial constraints:

$\forall$ var, val $\neg(\operatorname{Var}($ var, val $) \wedge$ val $\neq 0 \wedge$ val $\neq 1)$,

$\forall i d, v_{1}, x_{1}, v_{2}, x_{2}, v_{3}, x_{3} \neg\left(C l\left(i d, v_{1}, x_{1}, v_{2}, x_{2}, v_{3}, x_{3}\right) \wedge \operatorname{Var}\left(-, v_{1}, x_{1}^{\prime}\right) \wedge \operatorname{Var}\left(-, v_{2}, x_{2}^{\prime}\right)\right.$ $\left.\wedge \operatorname{Var}\left(-, v_{3}, x_{3}^{\prime}\right) \wedge x_{1} \neq x_{1}^{\prime} \wedge x_{2} \neq x_{2}^{\prime} \wedge x_{3} \neq x_{3}^{\prime} \wedge \operatorname{Dummy}(1)\right)$.

The last denial can be replaced by 8 denial constraints without inequalities considering all the combination of values for $x_{1}, x_{2}, x_{3}$ in $\{0,1\}$.

Assume now that $C_{1}, \ldots, C_{m}$ are the clauses in $\psi$. For each propositional variable $X_{i}$ store in table $\operatorname{Var}$ the tuple $\left(X_{i}, 0\right)$, with weight 1 , and $\left(X_{i}, 1\right)$ with weight $2^{n-i}$. Store tuple 1 in Dummy with weight $2^{n} \times 2$. For each clause $C_{i}=$ $l_{i_{1}} \vee l_{i_{2}} \vee l_{i_{3}}$, store in Clause the tuple $\left(C_{i}, X_{i_{1}}, \tilde{l}_{i_{1}}, X_{i_{2}}, \tilde{l}_{i_{2}}, X_{i_{3}}, \tilde{l}_{i_{3}}\right)$, where $\tilde{l}_{i_{j}}$ is equal to 1 in case of positive occurrence of variable $X_{i_{j}}$ in $C_{i}$; and to 0 , otherwise. For example, for $C_{6}=X_{6} \vee \neg X_{9} \vee X_{12}$, we store $\left(C_{6}, X_{6}, 1, X_{9}, 0, X_{12}, 1\right)$. The weight of this tuple is $2^{n}$.

Then the answer to the ground atomic query $\operatorname{Var}\left(X_{i}, 1\right)$ is yes iff the variable $X_{i}$ is assigned value 1 in the lexicographically maximum assignment (in case such a satisfying assignment exists). In case a satisfying assignment does not exist, then the tuple in Dummy has to be changed in order to satisfy the constraints. No attribute value in a tuple in Clause is changed, because the cost of such a change is higher than a change in the Dummy relation.

Proof of Lemma 4: If a vertex $v$ in $G$ has degree 2, then we transform it into a vertex of degree 4 by hanging from it an "ear" as shown in the figure, which is composed of three connected versions of the graph $H_{3}$ [20, Theorem 2.3] plus two interconnected versions of a box graph (c.f. figure below).

\footnotetext{
$\overline{{ }^{3} E \frac{E_{1}}{E_{2}} \text { means }}$ the expression obtained by replacing in expression $E$ the subexpression $E_{1}$ by expression $E_{2}$.
} 
It is easy to see that the "ear" is regular of degree 4, is 3-colorable (as shown in the picture with colors $r, g, b$ ), but not planar. Hanging the ear adds a constant number of vertices. Now we have to deal with the set $V_{\text {odd }}$ of vertices of degree 1 or 3 (vertices of degree 0 can be ignored). By Euler's theorem, $V_{\text {odd }}$ has an even cardinality. This makes it possible to pick up disjoint pairs $\left\{v_{1}, v_{2}\right\}$ of elements

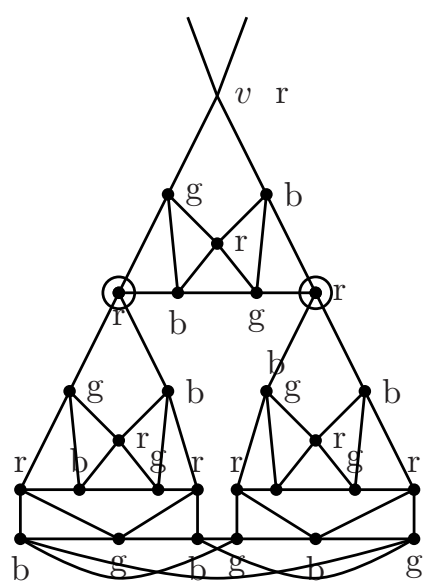

of $V_{\text {odd }}$, leaving every vertex coupled to some other vertex. For each such pair, $\left\{v_{1}, v_{2}\right\}$, add an extra vertex $v^{\prime}$ connected to (only) $v_{1}$ and $v_{2}$. This trio is 3 -colorable. Now $v_{1}, v_{2}$ have degree 2 or 4. From those that become of degree 2 , hang the "ear" as before. In this way, all the nodes become of degree 4 . The number of added vertices is polynomial in the size of the original graph. The 4-colorability of $G^{\prime}$ follows from the 4-colorability of $G$ (every planar graph is 4-colorable) and the 4-colorability of the hanging ears.

Proof of Corollary 3: From Lemma 4 and the $N P$-hardness of 3-colorability for planar graphs with vertices of degree at most 4 [20].

Proof of Theorem 4: If the update operation $U$ is a delete of a database atom, we reduce to our problem 3-Colorability of planar graphs $G$ with vertex degree at most 4 , which is $N P$-complete [20]. Given such a non-empty graph $G$, we construct graph $G^{\prime}$ as in Lemma 4, which is also 4-colorable (because $G$ is and the ears too).

Let $E(X, Y)$ be a database relation encoding the edges of the graph, Coloring a 2-ary database relation storing a coloring of the vertices, and Colors a unary relation storing the four colors allowed. Notice that a 4-coloring of $G$ can be found in polynomial time [32]. Then also a 4-coloring for $G^{\prime}$ can be found in polynomial time (a 4-coloring for the ears can be given once and for all). The ICs, essentially denials and inclusion dependencies, are as follows:

1. Every node is colored: $\forall x y \exists z(E(x, y) \rightarrow$ Coloring $(x, z))$.

2. Nodes have one color: $\forall x y_{1} y_{2} \neg\left(\right.$ Coloring $\left(x, y_{1}\right) \wedge$ Coloring $\left.\left(x, y_{2}\right) \wedge y_{1} \neq y_{2}\right)$.

3. Colors must be allowed: $\forall x y(\operatorname{Coloring}(x, y) \rightarrow \operatorname{Colors}(y))$.

4. Vertex degree is not less than 4: $\forall x\left(\exists y E(x, y) \rightarrow \exists y_{1} y_{2} y_{3} y_{4}\left(E\left(x, y_{1}\right) \wedge\right.\right.$ $E\left(x, y_{2}\right) \wedge E\left(x, y_{3}\right) \wedge E\left(x, y_{4}\right) \wedge y_{1} \neq y_{2} \wedge y_{1} \neq y_{3} \wedge y_{1} \neq y_{4} \wedge y_{2} \neq y_{3} \wedge y_{2} \neq$ $\left.\left.y_{3} \wedge y_{3} \neq y_{4}\right)\right)$.

5. Vertex degree is not bigger than 5: $\forall x y_{1} \cdots y_{5} \neg\left(E\left(x, y_{1}\right) \wedge \cdots \wedge E\left(x, y_{5}\right) \wedge y_{1} \neq\right.$ $\left.y_{2} \cdots \wedge y_{4} \neq y_{5}\right)$.

6. Only vertices are colored: $\forall x y \exists z(\operatorname{Coloring}(x, y) \rightarrow E(x, z))$.

7. All colors are used: $\forall x \exists z(\operatorname{Colors}(x) \rightarrow \operatorname{Coloring}(z, x))$. 
8. $E$ is symmetric: $\forall x y(E(x, y) \rightarrow E(y, x))$.

9. Adjacent vertices have different colors:

$\forall x y u w \neg(E(x, y) \wedge \operatorname{Coloring}(x, u) \wedge \operatorname{Coloring}(y, w) \wedge u=w)$.

The initial database $D$ stores the graph $G^{\prime}$, together with its 4-coloring (that does use all 4 colors). This is a consistent instance.

For the incremental part, if the update $U$ is the deletion of a color, e.g. delete $_{\text {Colors }}(c)$, i.e. of tuple $(c)$ from Colors, the instance becomes inconsistent, because an inadmissible color is being used in the coloring. Since repairs can be obtained by changing attribute values in existing tuples only, the only possible repairs are the 3 -colorings of $G^{\prime}$ with the 3 remaining colors (if such colorings exist), which are obtained by changing colors in the second attribute of Coloring. If there are no colorings, there are no repairs.

The query $Q$ : Colors $(c)$ ? is consistently true only in case there is no 3coloring of the original graph $G$, because it is true in the empty set of repairs.

Proof of Theorem 5: We reason basically as in the proof of theorem 4(c) in [6]; just introduce a new relation Dummy, and transform every denial $\forall \bar{y} \neg\left(A_{1} \wedge\right.$ $\left.\cdots \wedge A_{s}\right)$ there into $\forall \bar{y} \forall x \neg\left(A_{1} \wedge \cdots \wedge A_{s} \wedge\right.$ Dummy $\left.(x)\right)$. If we start with the empty extension for Dummy, the database is consistent. On the update part, if we insert the tuple Dummy(c) into the database, and the original denials were inconsistent in the given instance, then we cannot delete that tuple and no change in it can repair any violations. Thus, the only way to repair database is as in [6], which makes CQA $P^{N P}$-hard. 